\title{
A Novel Flexible PCB Conductive Structure for Electrodynamic Bearings and Measurement in its Induced Voltage
}

\author{
Guoping Ding ${ }^{\dagger}$, Jan Sandtner* and Hannes Bleuler**
}

\begin{abstract}
This paper proposes the concept of FlexPCB(flexible Printed Circuit Board) conductive structure for electrodynamic bearings. It has three main advantages: easy "printing" of considerably thin conductive wires, resulting in potential reduction in stray eddy currents; realization of specific conductive configurations with high precision to optimize the eddy current flowing; simplicity in being wound to cylinders or hollow cylinders of different diameters. To verify this new concept, the FlexPCB conductive structure was manufactured, an axial electrodynamic bearing test rig was built and the conductive structure's induced voltage was measured along the axial displacements from $0 \mathrm{~mm}$ to $56 \mathrm{~mm}$ at three rotating speeds. The finite element method was used to calcuatlate the flux density of electrodynamic bearing and induced voltage of the FlexPCB conductive structure. The experimental results are compared with the results from the FEM calculation. It is concluded that the measured and calculated induced voltages have consistency in the middle part of the bearing.
\end{abstract}

Keywords: Flexible PCB, Conductive structure, Electrodynamic bearings, Induced voltage, Finite element method

\section{Introduction}

Electrodynamic bearings (EDBs) are a type of magnetic bearing that can realize the contactless suspension based on the Lenz laws. The principle of electrodynamic bearings is based on on the motion of a conductor in a magnetic field. As this motion causes the variation in the magnetic flux linked to the conductor, a electromotive force generates, which induces eddy currents and in turn generates electromagnetic forces. This principle can be applied to the rotating systems, in which electromagnetic forces are generated to levitate the rotating part. Several kinds of electrodynamic bearings device are designed, aiming at providing the levitation of a rotor in axial or in radial direction.

For electrodynamic bearings, as the levitation can be obtainedin a passive way, they have more advantages compared to active magnetic bearings (AMBs): less complexity, higher reliability due to the lack of sensors and electronics and even lower cost [1-2]. Nevertheless, electrodynamic bearings have some drawbacks; for example, compared to active magnetic bearings, the achievable stiffness and damping are relatively low. Besides, as electrodynamic bearings enable the levitation only when the relative speed is above a threshold value, the stability at low speed is one challenge they face. In view of this, the

$\dagger$ Corresponding Author: School of Mechanical and Electronic Engineering, Wuhan University of Technology, China. (dgp7910@163.com)

* Silphenix GmbH, Switzerland. (jan.sandtner@eblcom.ch)

* Robotic Systems Laboratory, Ecole Polytechnique Fédérale de Lausanne(EPFL), Switzerland. (hannes.bleuler@epfl.ch)

Received: March 18, 2014; Accepted: April 21, 2015 back-up bearings are required for the operation at low speed or at rest. Furthermore, electrodynamic bearings do not allow any kind of tuning after being mounted, in contrast with AMBs which are easily tunable by acting on the control parameters.

The characteristics the electrodynamic bearings have make them a competitive alternative to classical AMBs in machines or applications, where the contactless nature of the levitation allows sacrificing the requirement of high radial stiffness. The earliest application of electrodynamic bearings dates back to the 1980s and 1990s — used for flywheels [3-15] because electrodynamic bearings' features matched flywheels' requirements very well. Firstly, flywheels typically work at the state of high-speed rotation, and it is at such high rotation speed that electrodynamic bearings can realize levitation; secondly, stiffness and rotation precision are not core requirements of flywheels, which are also drawbacks of electrodynamic bearings. Nowadays this type of rotor can be found in several high speed applications including flywheels, turbo-molecular pumps, small compressors, vacuum cleaners, high speed electric mandrels, etc.

Obviously, electrodynamic bearings offer the simplest means of magnetic levitation without electronics or cooling systems. The electrodynamic bearings' levitating force is generated from the interaction between magnetic field and eddy current. However, at the same time, the eddy current also causes power losses which has negative impact on the electrodynamic bearings application. In fact, in the nature of electrodynamic bearings levitation, the eddy currents are necessary but not always required, because only the azimuth eddy currents produce restoring forces while other 
eddy currents mainly contribute to the losses. Therefore, the conductor's configuration plays an important role in electrodynamic bearings operation as it will determine flow direction and distribution of eddy currents. Moreover, stray eddy currents inside the conductor also cause losses. So there are two requirements in the conductor design: firstly, the conductor's configuration should be optimized to increase the azimuth eddy currents while reducing other undesired eddy currents; secondly, the conductor should be considerably thin to reduce stray eddy currents and the related losses.

In the previous researches, the cylinder conductors and short-circuits coils were commonly used in the conductor configuration in EDBs [1, 9-14]. However, it is not sufficient for them to meet the above requirements. For conductive cylinders, it is difficult to lead the induced eddy currents into the desired current paths, and the stray eddy currents will have much less negative effect on losses owing to awful orientation of magnets. For short-circuited coils, it is difficult to for them to fully occupy the bearing space to produce azimuth eddy currents efficiently, so they are not suitable for high speed applications due to the centrifugal load on each discrete component.

In this paper, a new conductive structure is proposed, which is made of flexible PCB (flexible Printed Circuit Board, FlexPCB) for electrodynamic bearings. Comparing to common conductive structures mentioned above, it has some advantages. Firstly, it is easy to use FlexPCB to "print" very thin conductive wires, which can potentially reduce stray eddy currents. Secondly, it is easy to use FlexPCB to realize specific conductive configurations with high precision that optimizes the eddy current flowing. Thirdly, the FlexPCB can be easily wound to cylinder or hollow cylinder with different diameters. In this paper, in order to verify the performance of this novel FlexPCB conductive structure, an axial electrodynamic bearing test rig was built and its induced voltage was measured at various axial displacements and rotating speeds.

\section{FlexPCB Conductive Structure Used in the Cylindrical Axial Electrodyamic Bearings}

In this paper, the FlexPCB conductive structure is used in the axial cylindrical electrodynamic bearings. The configuration of axial cylindrical electrodynamic bearing is characterized by a rotating alternating magnetic field and a cylindrical conductor located on the static part of the system. A schematic representation of this configuration is shown in Fig. 1, where the rotation and axial movement of the magnetic field are shown. In the scheme, the conductor is represented by a set of coils.

Provided that the rotor remains at the axially nominal position as shown in Fig. 1(a), as one half of the magnetic flux coming from the magnet arrays and interacting with the coils goes upwards and the other half goes downwards, the flux cancels each other. Therefore, no macroscopic current is induced in the coils and no force arises. However, if the rotor is displaced from this nominal position, the voltage is induced within the coils. As the currents flow through each coil, an axial restoring force is created as shown in Fig. 1(b). In Fig. 1(b), only the azimuthal currents located within the radial magnetic field produce restoring forces. Since the azimuthal currents in each coil flow into the wires along the circumferential direction, the wires in circumferential direction dominantly contribute to the generation of restoring force while the wires in axial direction are only used to form the close circuit loop. Typically, the coils shown in Fig. 1(c) are of a kind of discrete distribution where the wires in the circumferential

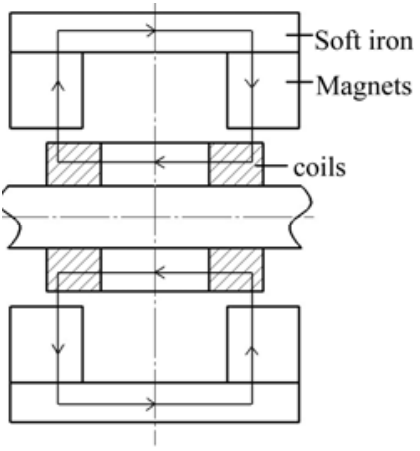

(a)

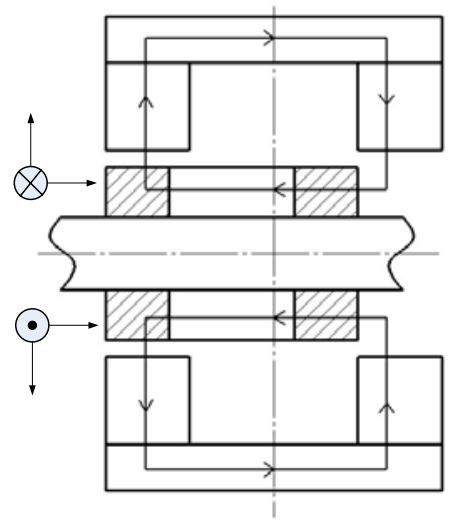

(b)

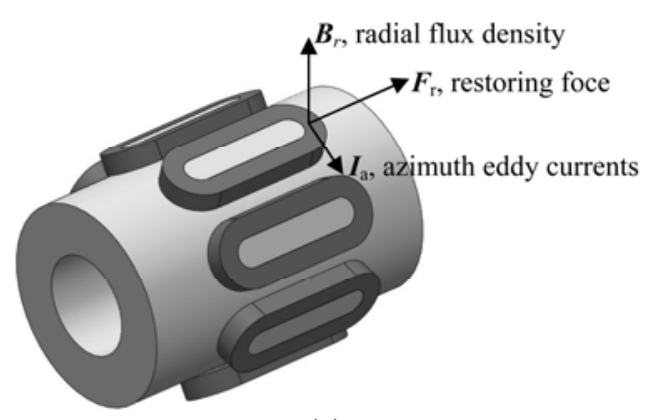

(c)

Fig. 1. A cylindrical axial electrodyamic bearing: (a) Nominal position; (b) Displaced position; (c) Discrete coils and restorting force. 


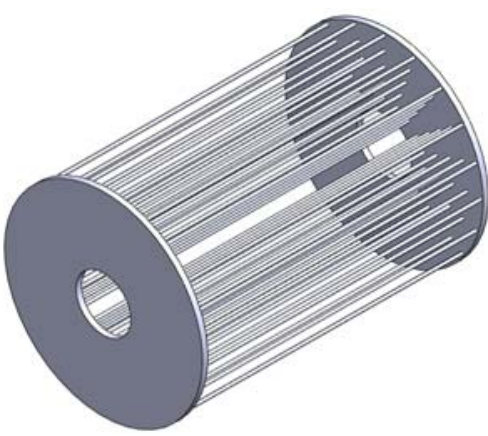

Fig. 2. Scheme of distributed coils.

direction of each coil are separated; in addition, the circumferential space between each coil is not utilized effectively. Furthermore, the discrete coils cause the pulsating restoring forces.

To solve these problems, a new conductive structure is proposed, which connects the wires in the circumferential direction (They are called "distributed coils") as shown in Fig. 2. The distributed coils are made of many thin insulated copper wire segments. The segments are oriented in the axial direction with several layers in radial direction; thus a cylindrical shape is formed. The segment ends are interconnected on the face ends of the cylinder in such a way that induced currents are forced to flow in rectangular loops covering the full length of the segments. This new structure is like a copper cylinder, which almost fully occupies the volume as much as possible. However, as it is made of many connected loops, it enables the eddy currents in the desired path to reduce losses and eliminate force ripple.

For the distributed coils, they must meet two requirements: (1) Each wire's thickness or diameter should be minimized, at least less than $0.3 \mathrm{~mm}$; (2) the spacing between each wire should be minimized, which means that wires should be as dense as possible in distribution. By doing this, some unnecessary stray eddy currents can be reduced and the space can be maximally occupied. Its manufacturing method can be seen in [14]. But the manufacturing is complicated and impractical, especially when the wires are required to be as thin as possible.

To meet the above requirements, an idea of realizing distributed coils through using flexible PCB(FlexPCB) is proposed, which can "print copper wires" on a FlexPCB and be wound to a cylinder as shown in Fig. 3(a). As is mentioned above, the FlexPCB conductive structure has these main advantages: the copper wires can be "printed" considerably narrowly and thinnly with high precision which benefits for reducing unnecessary eddy currents; it is very easy to print two wires connecting the parallel axial wires at two ends, which largely simplifies the manufacturing, since if common copper wires are used, an external copper plate was needed to be electroplated at two ends to connect the axial coils. Moreover, it was flexible to be wound to cylinders of different diameters.

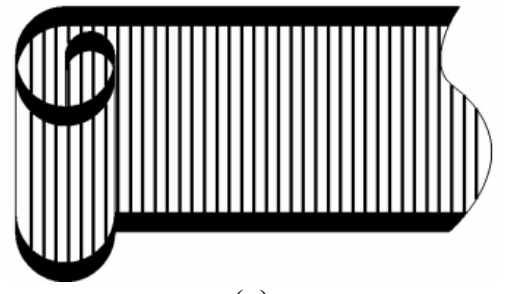

(a)

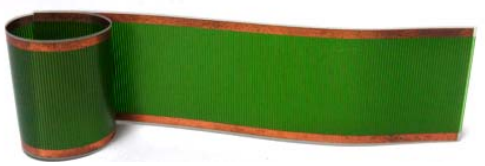

(b)

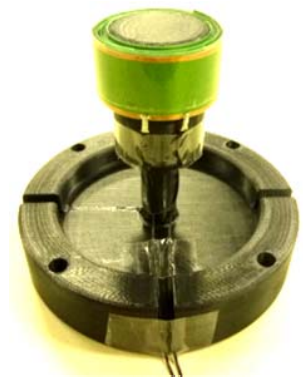

(c)

Fig. 3. FlexPCB conductive structure: (a) Scheme of FlexPCB; (b) A FlexPCB piece; (c) FlexPCB conductive structure

In the design of the FlexPCB conductive structure, its main geometric parameters are determined by the electrodynamic bearing's configuration, size, magnetic field distribution and the technological parameters of the FlexPCB process. Firstly, seen from the size of the FlexPCB film, it has these features: its thickness is $0.1 \mathrm{~mm}$, which is determined by the base material's thickness standard; as its length is limited by the manufacturing equipment we use, its processing length is no more than $100 \mathrm{~mm}$, and the length of each piece of the FlexPCB film is set $100 \mathrm{~mm}$ (In total, 25 pieces) as shown in Fig. 3(b); the width of the film is $20 \mathrm{~mm}$, which is determined by the optimal axial length of the magnetic circuit at the nominal position. Secondly, seen from the size of the "printed wire", it has these features: its thickness is $0.018 \mathrm{~mm}$; for each axially parallel wire, its width and the spacing between them are $0.3 \mathrm{~mm}$ and $0.15 \mathrm{~mm}$ respectively; the above parameters are considered in the FlexPCB manufacturing process; the width of connecting wires at two ends is $2 \mathrm{~mm}$ which is much larger than the width of the axially parallel wire with the consideration that the azimuth eddy currents flowed in the two connecting wires. Since the total axial width of the film is $20 \mathrm{~mm}$, the axial length of each axially parallel wire is $16 \mathrm{~mm}$. To efficiently use the FlexPCB film, the copper wires are "printed" on both sides of the film, so the total thickness of each FlexPCB piece is $0.136 \mathrm{~mm}$. The FlexPCB pieces are wound and glued on a plastic 
mandrel of diameter $32 \mathrm{~mm}$ as shown in Fig. 3(c). Since the electrodynamic bearing's inner diameter is $48 \mathrm{~mm}$, the FlexPCB assembly outer diameter is $44 \mathrm{~mm}$ to realize a $2 \mathrm{~mm}$ air gap between the bearing and the FlexPCB assembly.

The connecting copper on the outermost piece is exposed without insulating material, so it is convenient to connect external measurement circuits.

\section{Permanent Magnet Rotor}

The FlexPCB conductive structure works as the stator in the electrodynamic bearing test rig. The main rotating part is composed of a permanent magnets (PM) array with soft iron plates, as is shown in Fig. 4(a). The PM array has six

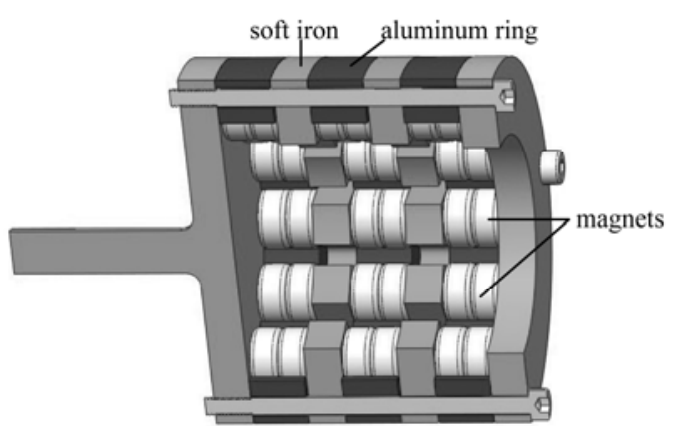

(a)

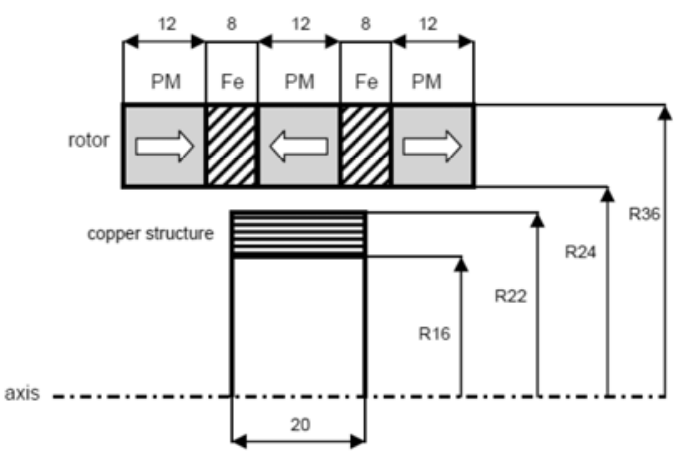

(b)

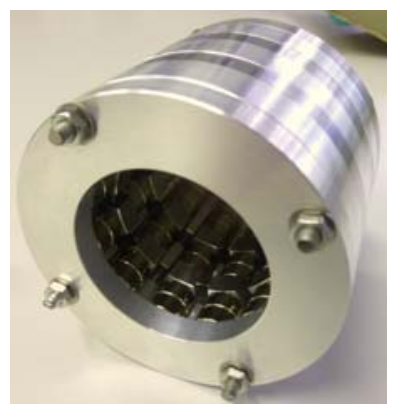

(c)

Fig. 4. Permanent magnet rotor: (a) Assembly of the permanent magnet rotor; (b) Flux of the permanent magnet rotor; (c) Picture of the permanent magnet rotor layers, each of which consists of 12 pieces of $\mathrm{NdFeB}$ (Neodymium Iron Boron) cylinder magnets purchased from Supermagnete company. The dimension of each magnet is $\Phi 12 \mathrm{~mm} \times 6 \mathrm{~mm}$, which is axially magnetised. The residual magnetism of the magnet is $1.32-1.37 \mathrm{~T}$, and the coercive field strength is $860-995 \mathrm{kA} / \mathrm{m}$. The magnets in each layer are embedded circumferentially in an aluminum ring to produce a NSNS alternative magnetic field along the perimeter. Two soft iron rings are sandwiched between six layers of magnets to guide the flux lines, as is shown in Fig. 4(b). The cover and the flange of each end are made of stainless steel. The cover, aluminum rings with magnets arrays, soft iron rings and flange are connected and fastened through four screws. The outer and inner diameters of the rotating assembly are $80 \mathrm{~mm}$ and $48 \mathrm{~mm}$ respectively. The assembly of rotor is shown in Fig. 4(c).

To investigate the magnetic field of axial electrodynamic bearing, the finite element method(FEM) is used to calculate the flux density and flux. In the FEM calculation, the finite element model is first constructed as shown in Fig. 5. Then the materials' properties, including the relative magnetic permeability of permanent magnet, soft iron, stainless steel and air, and the permanent magnet's residual magnetism and coercive field strength are applied in their corresponding area. Finally a zero-magnetic vector potential as the boundary condition indicating static field is applied at the exterior of the air layer. The model is solved

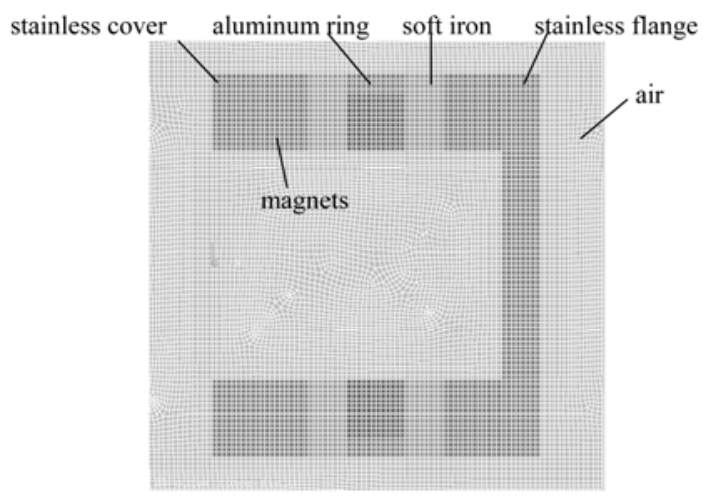

Fig. 5. Finite element model of the aixial electrodynamic bearing

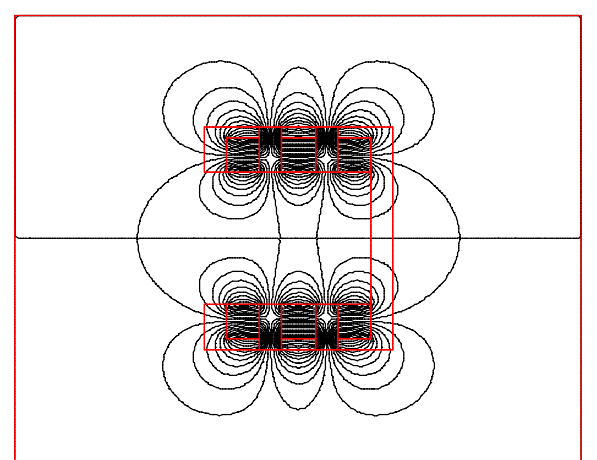

Fig. 6. Flux lines of the aixial electrodynamic bearing 


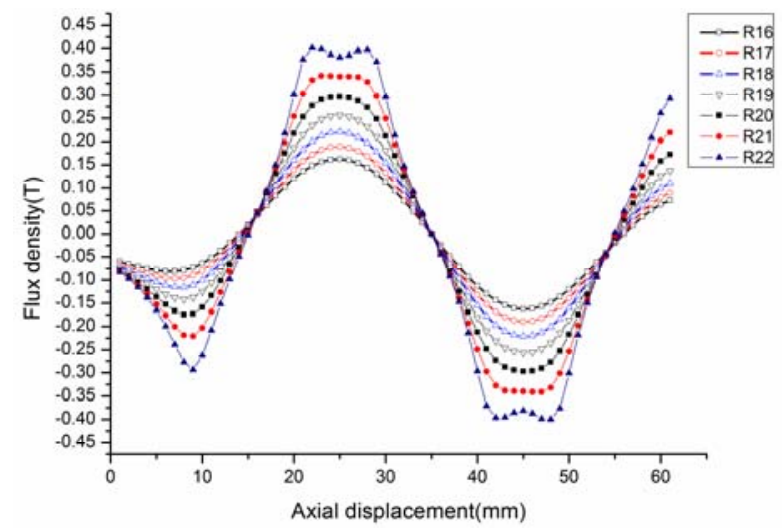

Fig. 7. Flux density along the axial displacement at the radius of from $16 \mathrm{~mm}$ to $22 \mathrm{~mm}$

and the flux lines are shown in Fig. 6. Fig. 7 shows the flux density along the axial displacement at the radius of from $16 \mathrm{~mm}$ to $22 \mathrm{~mm}$. The initial axial point is at the left edge of stainless cover in Fig. 5. The FlexPCB conductive structure has the radius of from $16 \mathrm{~mm}$ to $22 \mathrm{~cm}$; Fig. 7 shows the flux density distribution at the conductive structure' location, which is used for the flux calculation.

\section{Induced Voltage Measurement of the FlexPCB Conductive Structure}

To verify the feasibility of the FlexPCB conductive structure, the induced voltages at various axial displacements and rotating speeds are measured and analyzed. In the test rig, the FlexPCB conductive structure and the PM array rotor are assembled as a kind of configuration of inner stator and outer rotor. Since the FlexPCB conductive structure is static, it is practicable to measure its induced voltage. The PM rotor is mounted on a lathe's spindle and the FlexPCB structure with its base is clamped by the chuck of the tailstock, as is shown in Fig. 8. Such a device guarantees the concentricity of the PM rotor and the FlexPCB structure. The air gap between the PM array and the FlexPCB structure is $2 \mathrm{~mm}$. An external circuit is connected with the outermost FlexPCB piece to measure the induced voltage on it, and the induced voltage is recorded by a NI-6280 data acquisition card.

In the measurement, the rear-end surface of the PM rotor and the front-end surface of the FlexPCB structure are first aligned, which is the initial position. In addition, the lathe's spindle is started to drive the PM rotor at a certain speed, and then the induced voltage is acquired. After the acquisition of induced voltage at one axial position is completed, the tailstock is moved to insert the FlexPCB structure into the PM rotor, and the data acquisition is repeated. The axial increment of each movement is $1.0 \mathrm{~mm}$, and the total axial movement is $58 \mathrm{~mm}$. After the acquisition of induced voltage at the whole axial displacement is completed, the FlexPCB structure is pulled out to the initial

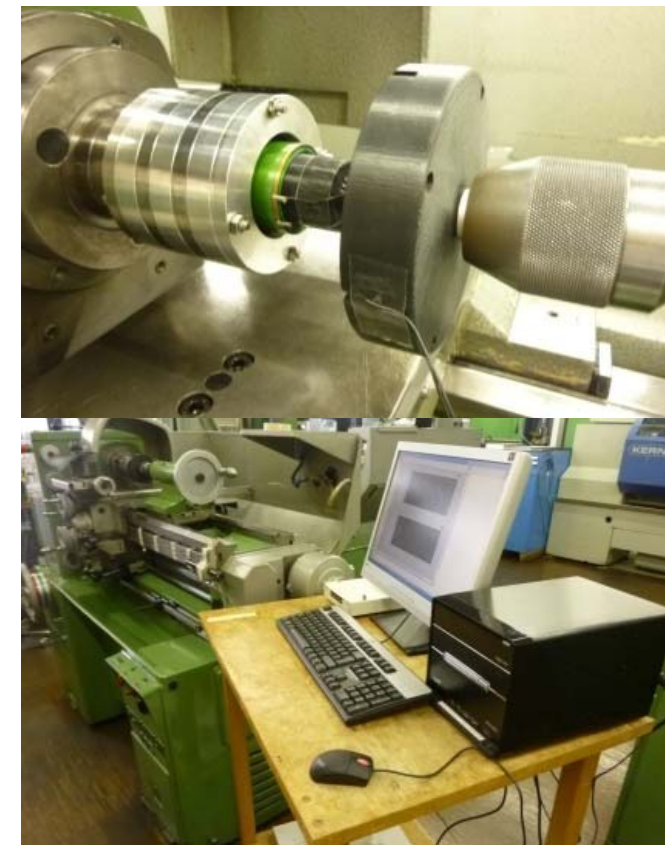

Fig. 8. Induced voltage measurement

axial position and the spindle is set at another speed, and the above procedures are repeated. Three rotating speeds are set in the measurement: 1000rpm, 1500rpm and $2000 \mathrm{rpm}$.

Fig. 9(a) shows the induced voltage at therotating speed of $2000 \mathrm{rpm}$ and the axial displacement of $10 \mathrm{~mm}$; Fig. 9(b) shows the FFT analysis of induced voltages at 3 kinds of rotating speeds and axial displacement of $10 \mathrm{~mm}$; and Fig. 9(c) shows the amplitude of induced voltages along total axial displacement at 3 kinds of rotating speeds.

In Fig. 9(a), the curve of induced voltage with the change of time is virtually harmonic, which is consistent with prediction that the induced voltage at a certain place along the axial direction varies with the flux variation caused by magnets rotation. At a given rotation speed, the induced voltage's frequency is a constant value.

In Fig. 9(b), for the axial displacement of $10 \mathrm{~mm}$, the frequency at three kinds of rotating speed of $1000 \mathrm{rpm}$, $1500 \mathrm{rpm}$ and $2000 \mathrm{rpm}$ is $87 \mathrm{~Hz}, 140 \mathrm{~Hz}$ and $185 \mathrm{~Hz}$ respectively, and amplitude of induced voltage is $0.60 \times$ $10^{-3} \mathrm{~V}, 0.82 \times 10^{-3} \mathrm{~V}$ and $1.35 \times 10^{-3} \mathrm{~V}$ respectively. Since the PM rotor has 6 magnet poles, the frequency of flux variation at three kinds of rotating speed of $1000 \mathrm{rpm}$, $1500 \mathrm{rpm}$ and $2000 \mathrm{rpm}$ is $100 \mathrm{~Hz}, 150 \mathrm{~Hz}$ and $200 \mathrm{~Hz}$ respectively. Theorically, the frequency of the induced voltage should be equal to the one of the flux variation. The measurement results are almost consistent with the theoretical analysis, but there is a $10 \%$ error, which is possibly caused by the rotation speed error of the lathe. The amplitude of induced voltage increases virtually and linealy with the rotation speed, which is consistent with the theoretical prediction.

Fig. 9(c) shows the induced voltage measured and 


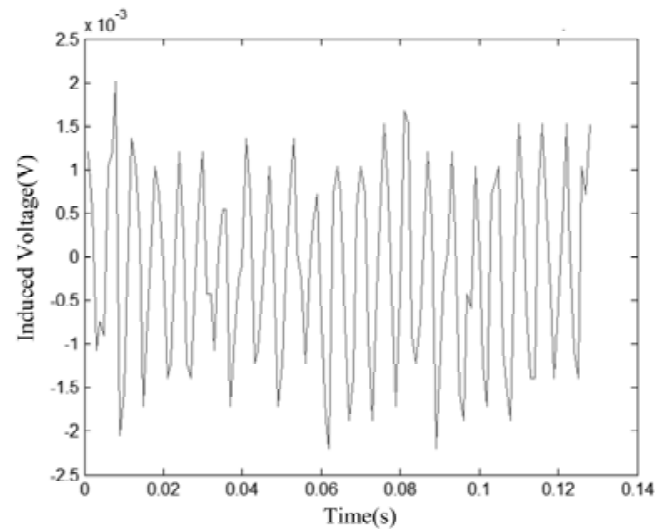

(a)

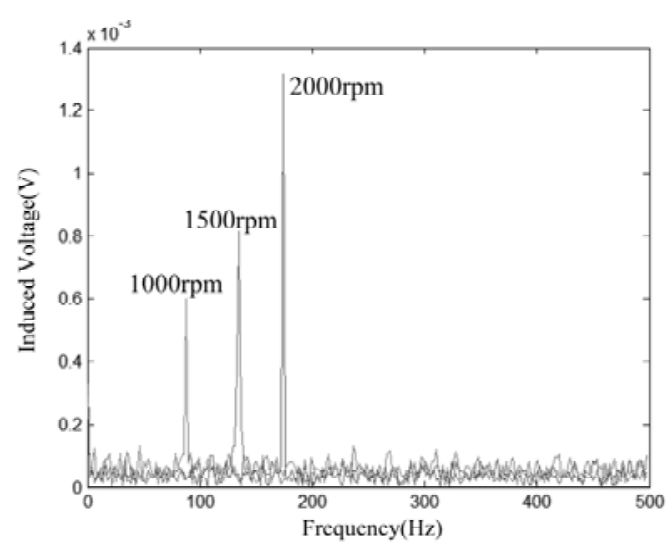

(b)

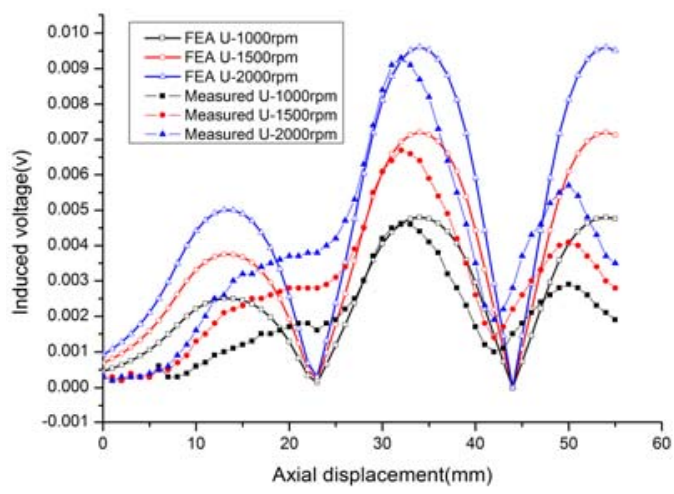

(c)

Fig. 9. Measured Induced voltage: (a) Induced voltage at the rotating speed of 2000rpm and axial displacement of 10mm; (b) FFT analysis of the induced voltages at 3 kinds of rotating speeds andaxial displacement of 10mm; (c) Amplitude of induced voltages along total axial displacement at 3 kinds of rotating speeds

through the FEM calculation. Fig. 9(c) shows the curves of measured induced voltage amplitudes at three kinds of rotation speeds along the whole axial displacement, indicating similar variation trend between their magnitudes scaled and rotation speeds. At each rotation speed, the measured induced voltage amplitude increases first with the axial displacement. At the axial displacement of $33 \mathrm{~mm}$, the measured induced voltage amplitude reaches the maximum value. And then, while the conductive structure's axial displacement continually increases, the induced voltage amplitude begins to drop. At the axial displacement of $43 \mathrm{~mm}$, the induced voltage amplitude decrease to the minimum value. Hence, here the opposite fluxes almost cancel each other. After the conductive structure passes the lowest net flux point, the induced voltage amplitude begins to increase.

To further investigate the amplitude of induced voltage, the induced voltage is calculated theoretically based on the following formula:

$$
U=\omega * n * \Phi
$$

In this formula, $U$ is induced voltage, $\omega$ is the angular frequency of variation of magnetic field, $n$ is the turns of the coil and $\Phi$ is the flux linked to the conductor.

The flux $\Phi$ along axial displacement at the radius of $22 \mathrm{~mm}$ (i.e. the FlexPCB structure's upper boundary) is retrieved from the FEM calculation.

In Fig. 9(c), the FEM-calculated induced voltages can be divided into three wave segments. In the first wave, the FEM-calculated induced voltage increases with the axial displacement increment; at the axial displacement of $13 \mathrm{~mm}$, after it reaches the maximum value, it begins to decrease and to the minimum value at $23 \mathrm{~mm}$. In the second wave, the induced voltage reaches the maximum value and minimum value at $34 \mathrm{~mm}$ and $45 \mathrm{~mm}$ respectively. In the third wave, the induced voltage reaches the maximum value at $53 \mathrm{~mm}$. These three waves's minimum values are all almost near zero. The first wave's peak value is less than those of the second wave and third wave, but the latter two are equal.

The curve of calculated induced voltages is consistent with the magnetic field distribution of the electrodynamic bearing as shown in Fig. 6. It indicates that, when the FlexPCB structure moves to the center of magnets, the radial flux cancels each other, and the net flux and induced voltage decrease to zero. In addition, when the FlexPCB structure located at the center of the stainless cover and soft iron, the radial flux reaches the maximum value, and the induced voltage also reaches the maximum value. Since the flux density near the stainless cover is smaller than the one of soft iron, the flux and induced voltage show the similar trend, which is the main reason that the first wave's peak value is less than those of the second wave and the third wave.

Comparing the measured and calculated induced voltage, they show the consistency only in the second wave. However, in the first and third waves, their differences are obvious. The main possible reason is that the electrodynamic bearing is mounted on the lathe to perform the induced voltage measurement. As most of the lathe's components are magnetic conductive materials, they may disturb the 
magnetic field and change the flux distribution of electrodynamic bearing.

\section{Conclusions}

In this paper, the concept of FlexPCB conductive structure for electrodynamic bearings is proposed. It main has three advantages: easy "printing" of considerably thin conductive wires, resulting in the reduction in stray eddy currents; realization of specific conductive configurations with high precision to optimize the eddy current flowing; simplicity in being wound to cylinders or hollow cylinders with different diameters. To verify this new concept, the FlexPCB conductive structure was manufactured, an axial electrodynamic bearing test rig was built and the conductive structure's induced voltage was measured along the axial displacements of from $0 \mathrm{~mm}$ to $56 \mathrm{~mm}$ at three rotating speeds. The finite element method was used to calcuatlate the flux density of electrodynamic bearing and induced voltage of the FlexPCB conductive structure. The experimentalresults are compared with the results from the FEM calculation. It is concluded that the measured and calculated induced voltages have consistency in the middle part of the bearing.

\section{Acknowledgements}

This work was supported by the National Science Foundation of China (Project No. 51105285).

\section{References}

[1] Torbjörn A. Lembke, "Design and Analysis of a Novel Low Loss Homopolar Electrodynamic Bearing", PhD Thesis, KTH Elec. Eng. Dept., Stockholm, 2005.

[2] Impinna Fabrizio, "Electrodynamic Bearings Modeling and Design", PhD Thesis, POLITECNICO DI TORINO, Torino, 2010.

[3] Paul Alan Basore, "Passive Stabilization of Flywheel Magnetic Bearings", Master Thesis, Massachusetts Institute of Technology, Cambridge, 1980.

[4] Richard F. Post, "Passive Magnetic Bearings for Vehicular Electromechanical Batteries", Report of Lawrence Livermore National Laboratory, 1996.

[5] R. F. Post, D. D. Ryutov and J. R. Smith, "Research on Ambient-Temperature Passive Magnetic Bearings at the Lawrence Livermore National Laboratory", in Proceedings of MAG '97 Industrial Conference, Exhibition and Short Course on Magnetic Bearings, Alexandria, VA, August 19-22, 1997.

[6] R. F. Post and D. D. Ryutov, "Ambient-Temperature Passive Magnetic Bearings: Theory and Design Equ- ations", in Proceedings of the 6th International Symposium on Magnetic Bearings, Cambridge, Massachusetts, August 5-7, 1998.

[7] R.F. Post and D.A. Bender, "Ambient-Temperature Passive Magnetic Bearings for Flywheel Energy Storage Systems", in Proceedings of the 7th International Symposium on Magnetic Bearings, Zurich, Switzerland, August 23-25, 2000.

[8] Richard F. Post, "Stability Issues in Ambient-Temperature Passive Magnetic Bearing Systems", Report of Lawrence Livermore National Laboratory, 2000.

[9] Alexei V. Filatov and Eric H. Maslen, "Passive Magnetic Bearing for Flywheel Energy Storage Systems", IEEE TRANSACTIONS ON MAGNETICS, vol. 37, no. 6, pp. 3913-3924, 2001.

[10] Alexei V. Filatov and Eric H. Maslen, "A method of noncontact suspension of rotating bodies using electromagnetic forces", JOURNAL OF APPLIED PHYSICS, vol.91, no.4, pp.2355-2371, 2002.

[11] Alexei V. Filatov and Eric H. Maslen, "Stability of an electrodynamic suspension", JOURNAL OF APPLIED PHYSICS, vol. 92, nod. 6, pp. 2355-2371, 2002.

[12] Jan Sandtner and Hannes Bleuler, "Electrodynamic Passive Magnetic Bearing with Planar Halbach Arrays", in Proceedings of the 9th International Symposium on Magnetic Bearings, Lexington, Kentucky, USA, August 3-6, 2004.

[13] Alexei V. Filatov and P. M., Kent Davey, "Richard Thompson.: Flywheel Energy Storage System with Homopolar Electrodynamic Magnetic Bearing", in Proceedings of the 10th International Symposium on Magnetic Bearings, Martigny, Switzerland, August 21-23, 2006.

[14] Jan Sandtner and Hannes Bleuler, "Compact Passive Electrodynamice Thrust Bearing", in Proceedings of the $11^{\text {th }}$ International Symposium on Magnetic Bearings, Nara, Japan, August 26-29, 2008.

[15] Nicola Amati, X. D. L. and Andrea Tonoli, "Modeling of Electrodynamic Bearings", Journal of Vibration and Acoustics, vol. 130, no. 9, p.2355-2371. p. 061007-1-9, 2008.

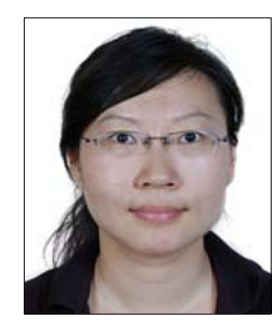

Guoping Ding She received her B.S. M.S. and Ph.D. degree from Wuhan University of Technology, Wuhan, China, all in Mechanical Engineering, in 2001, 2004 and 2008 respectively. She is working as Associate Professor in the School of Mechanical and Electronic Engineering of Wuhan University of Technology from 2011. Her research interests are about magnetic bearings, magnetic field simulation and sensors. 


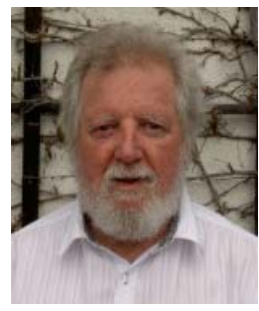

Jan Sandtner $\mathrm{He}$ received M.S. degree in Automation and Measuring technique from Czech Technical University, Prague, Czechoslovakia, in 1960. He is a Consultant in Robotic Systems Laboratory, Ecole Polytechnique Fédérale de Lausanne (EPFL), Switzerland. His research interests are about magnetic bearings and energy storage.

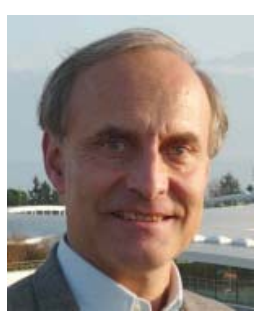

Hannes Bleuler $\mathrm{He}$ received M.S. degree in Electrical Engineering and Ph.D. degree in Mechatronics from ETH Zurich, Zurich, Switzerland, in 1978 and 1984 respectively. He is the full professor in Robotic Systems Laboratory, Ecole Polytechnique Fédérale de Lausanne (EPFL), Switzerland from 1995. His research interests are about magnetic bearings, microrobotics and biomedical robotics. 